OPEN ACCESS

Edited by:

Yu Xiang George Kong, The Royal Victorian Eye \& Ear

Hospital, Australia

Reviewed by:

Jianzhang $\mathrm{Hu}$,

Fujian Medical University, China

Yi Shao,

Nanchang University, China

*Correspondence:

Saiqun Li

123213197@qq.com

Jin Yuan

yuanjincornea@126.com

${ }^{\dagger}$ These authors have contributed equally to this work and share first authorship

Specialty section

This article was submitted to

Ophthalmology,

a section of the journal

Frontiers in Medicine

Received: 02 October 2021 Accepted: 03 November 2021 Published: 24 November 2021

Citation:

Wang Q, Yu F, Feng Z, Li W, Li N,

Du X, Zhao X, Li S and Yuan J (2021)

Changes in Anterior and Posterior Corneal Elevation in Patients With

Allergic Conjunctivitis

Front. Med. 8:788302.

doi: 10.3389/fmed.2021.788302

\section{Changes in Anterior and Posterior Corneal Elevation in Patients With Allergic Conjunctivitis}

\author{
Qian Wang ${ }^{1+}$, Fei $Y u^{1+}$, Ziqing Feng ${ }^{1}$, Weihua $L i^{1}$, Naiyang $L^{2}{ }^{2}$, Xinyue $D u^{1}$, Xuan Zhao ${ }^{1}$, \\ Saiqun $\mathrm{Li}^{1 *}$ and Jin Yuan ${ }^{1 *}$ \\ ${ }^{1}$ State Key Laboratory of Ophthalmology, Zhongshan Ophthalmic Center, Sun Yat-sen University, Guangdong Provincial Key \\ Laboratory of Ophthalmology and Visual Science, Guangdong Provincial Clinical Research Center for Ocular Diseases, \\ Guangzhou, China, ${ }^{2}$ Eye Center, Zhongshan City People's Hospital, Zhongshan, China
}

Purpose: To evaluate corneal elevation changes in patients with allergic conjunctivitis (AC) and to analyze their correlations with ocular allergy signs and corneal biomechanical alterations.

Methods: Thirty patients (30 eyes) with AC and twenty normal subjects (20 eyes) were included in this prospective study. All participants underwent a complete ocular examination, including corneal tomography by Pentacam and corneal biomechanics evaluation by Corvis ST. AC patients were evaluated for their eye rubbing frequency and ocular allergic signs.

Results: The elevation at the thinnest location (TE) and the central location (CE), the elevation difference at the thinnest location (TED) and the central location (CED), and the mean value of elevation difference in the central $4 \mathrm{~mm}$ zoom (MED) of both the anterior and posterior corneal surface were significantly higher in the AC group than in the normal group ( $p<0.05$ for all). In AC patients, only anterior corneal elevation parameters were positively correlated with eye rubbing frequency and ocular allergy sign severity $(p<0.05$ for all), while the tomography and biomechanical index (TBI) was positively correlated with the elevation parameters of both the anterior and posterior corneal surfaces $p<0.05$ for all).

Conclusion: AC patients carry an increased risk of corneal ectasia. Posterior corneal elevation parameters are sensitive and reliable predictors of keratoconus (KC) risk in AC patients.

Clinical Trial Registration: https://clinicaltrials.gov/ct2/show/NCT04299399, identifier [NCT04299399].

Keywords: allergic conjunctivitis, corneal tomography, corneal surface elevation, corneal posterior surface, corneal biomechanics

\section{INTRODUCTION}

Allergic conjunctivitis (AC) is a common ocular surface disease that affects more than $20 \%$ of the population (1). It is well established in the literature that $\mathrm{AC}$ is closely related to the occurrence of keratoconus (KC) (2-4). Various allergic ocular conditions, including vernal keratoconjunctivitis (VKC), atopic keratoconjunctivitis (AKC) and seasonal allergic conjunctivitis (SAC) or perennial 
allergic conjunctivitis (PAC), have been reported to increase the risk of developing KC (5). Although the underlying mechanisms remain unclear, increased inflammatory molecules and proteases and itch-provoked eye rubbing were thought to contribute to the development and progression of KC in AC patients (6).

$\mathrm{AC}$ has been found to be positively associated with early unset of KC. However, this association may still be underestimated in clinical analysis, since a considerable number of AC patients have subclinical presentation of $\mathrm{KC}$ (7). This subclinical population needs close monitoring for $\mathrm{KC}$ development. KC is characterized by progressive corneal thinning and a coneshaped protrusion. It can be easily diagnosed in its intermediate to advanced stages, but an exact diagnosis of subclinical $\mathrm{KC}$ is still a major challenge because the diagnostic criteria remain to be defined (8). Several corneal topographic parameters, such as corneal elevation parameters and corneal thickness distribution indices, have been evaluated for their sensitivity to subclinical KC (9-14). Previous studies have shown that among the measured topographic parameters, including pachymetric parameters, elevation parameters and topometric parameters, anterior and posterior elevations had the greatest areas under the receiver operating characteristic curve (AUROCs) to discriminate subclinical KC from normal corneas $(10,13)$. KC was thought to start from the posterior surface of the cornea $(15,16)$. Moreover, taking into consideration that compared to the anterior corneal surface, the posterior cornea surface is less affected by corneal epithelial conditions and tear film stability, previous studies proposed posterior corneal elevations as more sensitive and reliable shape parameters for differentiating subclinical KC $(15,16)$.

Many studies have screened KC-like topographic and biomechanical changes in AC patients. KC-like tomography was observed in $11-20 \%$ of VKC patients $(7,17)$. Moreover, VKC was reported to cause a reduction in corneal biomechanics, as indicated by decreased corneal resistance factor (CRF) (18). Additionally, our previous study found alterations in the tomography and biomechanical index (TBI) in AC patients (19). In terms of posterior corneal surface changes in AC patients, studies have mainly concentrated on corneal aberrations and densitometry, and few have investigated posterior surface elevations. Barreto et al. (20) reported that VKC patients had significantly higher anterior and posterior elevations and pachymeter indices than healthy subjects. However, this study used Orbscan tomography IIz, which has been questioned by some researchers about its ability to accurately measure the posterior corneal surface. Because the Orbscan topographer works on the principle of slit scanning combined with Placido-disk technology, the posterior elevation map is derived mathematically from the Placido-disk reflection and 20 slit scans of the anterior segment $(11,14)$. In contrast, Pentacam uses a rotating Scheimpflug camera to capture 25 slit images and obtain a representation of the corneal shape, with which the posterior corneal elevations can be measured with high reproducibility and repeatability $(21,22)$.

In this study, we evaluated the corneal tomographic changes in AC patients using Pentacam, focusing on anterior and posterior surface elevation and pachymetric distribution. The correlations of these corneal tomographic changes with ocular allergic signs and corneal biomechanical changes were further analyzed. The purpose of this study was to elucidate the risk of KC development in $\mathrm{AC}$ patients and to identify sensitive indicators to screen high-risk patients for early intervention.

\section{MATERIALS AND METHODS}

\section{Participants}

This prospective case-control study was conducted at the Zhongshan Ophthalmic Center, Sun Yat-Sen University and was approved by the Ethics Committee of the study hospital (2020KYPJ008). This trial has been registered at ClinicalTrials.gov as trial number NCT04299399. All subjects or responsible relatives signed informed consent forms before the study.

In this study, thirty AC patients and twenty normal subjects were included. The diagnosis of AC was determined according to the presence of typical clinical manifestations, including itching, redness, conjunctival hyperemia, palpebral conjunctival papillae or Horner-Trantas dots. Only patients with a history of AC for more than 2 years were enrolled. Age-matched subjects with no remarkable medical or ocular history except for refractive error served as normal controls. The exclusion criteria were: high refractive errors (spherical equivalent $>6 \mathrm{D}$ and/or astigmatism $>2 \mathrm{D}$ ), active ocular inflammatory diseases other than AC, previous ocular surgery or disease, systemic diseases that might lead corneal abnormalities, soft contact lens wearing within 2 weeks and rigid contact lens wearing within 1 month.

\section{Examination Methods}

Eye rubbing frequency and ocular allergy signs were evaluated in AC patients as reported in our previous study (19). Briefly, eye rubbing frequency was assessed on a scale from 1 (absent) to 5 (constant eye rubbing). Each objective ocular allergy sign (including conjunctival hyperemia, swelling, papillae, and corneal epithelial disorder) was graded on a scale from 0 (none) to 3 (severe) by the clinician.

All participants underwent a complete ophthalmic evaluation, including slit lamp examination, corneal tomography with Pentacam (Oculus, Inc., Wetzlar, Germany) and corneal biomechanics evaluation with Corneal Visualization Scheimpflug Technology (Corvis ST) (Oculus, Inc., Wetzlar, Germany). All measurements were performed by a single examiner to minimize interobserver variation.

The following Pentacam parameters were analyzed: (1) topographic parameters of the anterior and posterior corneal surface, including keratometric values [flat keratometry (K1), steep keratometry (K2), mean keratometry (Kmean), maximum keratometry (Kmax)], astigmatism and Q value; (2) Pachymetric parameters, including central and thinnest corneal thickness (CCT and TCT), pachymetric progression indices [maximum (PImax), minimum (PImin) and average (PIavg)], and Ambrosio relational thickness indices [maximum (ARTmax) minimum (ARTmin) and average (ARTavg)]. On the Belin/Ambrósio enhanced ectasia display, pachymetric progression indices (PPIs) refer to the rate of corneal thickness changes along each meridian 
starting from the thinnest corneal point. The maximum, minimum and average PPI are recorded. Relational thickness indices express the ratio of the TCT to each of the above three PPIs, as expressed in the following formulas: ARTavg = TCT/PIavg; ARTmin = TCT/PImin; ARTmax = TCT/PImax (16); and (3) Anterior and posterior corneal elevations, including elevations at the thinnest and central locations (TE and CE), mean elevation in the $4 \mathrm{~mm}$ central zone (ME), elevation differences at the thinnest and central locations (TED and CED) and in the central $4 \mathrm{~mm}$ zone (MED). Corneal elevations were defined as the distances between the corneal surface and the best-fit sphere (BFS) reference surfaces set at 8 -mm diameter. Corneal elevation difference values were taken as the differential changes in corneal elevation values between the BFS and the enhanced BFS (with exclusion of a 3.5- $\mathrm{mm}$ optical zone in the thinnest portion of the cornea) obtained with Belin/Ambrósio enhanced ectasia display software. An example of the data output for corneal elevations assessment is shown in Figure 1. The corneal biomechanical parameters measured by Corvis ST have been presented in detail in our previous article (19). TBI was selected for analysis in this study because we previously found that only TBI showed significant differences between AC patients and normal subjects.

\section{Statistical Analysis}

To prevent bias, one eye of each participant was selected for analysis (for AC patients, the eye with more severe AC symptoms or signs was selected; for normal subjects, the eye was randomly selected). Statistical analyses were performed using SPSS software (version 25.0; SPSS, Inc., Chicago, Illinois, USA). The normality of the continuous variables was evaluated by the Kolmogorov-Smirnov test. Comparisons of continuous data between groups were performed by applying independentsample $t$-tests. Categorical variables were compared using the $\chi^{2}$ test. Correlations between the continuous and ranked data were analyzed by Spearman's correlation test. $p<0.05$ was considered statistically significant.

\section{RESULTS}

\section{Patient Characteristics}

Twenty eyes of 20 normal subjects and thirty eyes of 30 AC patients were included in this prospective study. The demographics of the study population are shown in Table 1. There were no significant differences in age, sex, intraocular pressure (IOP) (measured by Corvis ST), spherical power (S), cylinder power $(\mathrm{C})$ or spherical equivalent (SE) between the two groups $(p>0.05$ for all).

\section{Corneal Topographic Parameters}

Comparisons of the main corneal topographic parameters between the normal and AC groups are shown in Table 2. We found no significant differences in terms of the anterior corneal surface parameters between the two groups $(p>0.05$ for all). The astigmatism and the $\mathrm{Q}$ value of the posterior corneal surface were significantly higher in the AC group than in the normal group ( $p=0.02$ and $p=0.01$, respectively). The remaining topographic parameters of the posterior surface were not significantly different between the two groups $(p>0.05$ for all).

\section{Corneal Pachymetric Parameters}

Comparisons of the main corneal pachymetric parameters between the normal and AC groups are presented in Table 3. No significant difference was observed in any of the pachymetric parameters between the two groups ( $p>0.05$ for all).

\section{Corneal Elevation Parameters}

Comparisons of the corneal elevation parameters of both the anterior and posterior corneal surfaces between the normal and AC groups are shown in Table 4. The TE, CE, TED, CED, and MED of both the anterior and posterior corneal surfaces were significantly higher in the AC group than in the normal group $(p<0.05$ for all $)$.

\section{Correlation of Corneal Elevation Parameters With Eye Rubbing Frequency and Ocular Allergy Sign Severity in AC Patients}

The correlations of the corneal elevation parameters with eye rubbing frequency and ocular allergy sign severity in AC patients are presented in Table 5. The anterior corneal surface elevation parameters were positively related to eye rubbing frequency and the severity of ocular allergy signs, including conjunctival swelling, papillae and epithelial disorder $(p<0.05$ for all). However, there was no correlation between the posterior corneal surface elevation parameters and eye rubbing frequency or ocular allergy sign severity ( $p>0.05$ for all).

\section{Correlation of Corneal Elevation Parameters With the TBI in AC Patients}

Table 6 shows the correlations of the corneal elevation parameters with TBI in AC patients. We found that TBI was positively correlated with TE $(\mathrm{r}=0.608, p<0.001), \mathrm{CE}(\mathrm{r}$ $=0.464, p=0.010)$, CED $(\mathrm{r}=0.727, p<0.001)$, and MED $(\mathrm{r}=$ $0.750, p<0.001)$ of the anterior corneal surface, as well as with $\mathrm{TE}(\mathrm{r}=0.563, p=0.001)$ of the posterior corneal surface.

\section{DISCUSSION}

A close association between $\mathrm{KC}$ and $\mathrm{AC}$ has been well established in the previous literature (23-25). However, sensitive indicators for screening $\mathrm{AC}$ patients at high risk of developing $\mathrm{KC}$ are still under investigation. In our previous study, we showed that TBI could be used as an indicator of $\mathrm{KC}$ development risk in AC patients (19). In the current study, we found that both the anterior and posterior corneal elevation values were significantly higher in AC patients than in normal subjects and were further identified to positively correlate with the altered TBI in AC patients. Our findings provide additional evidence supporting that compared to normal subjects, AC patients have an increased risk of corneal ectasia, and corneal elevation could be used as a sensitive indicator of the risk of corneal ectasia in AC patients. 


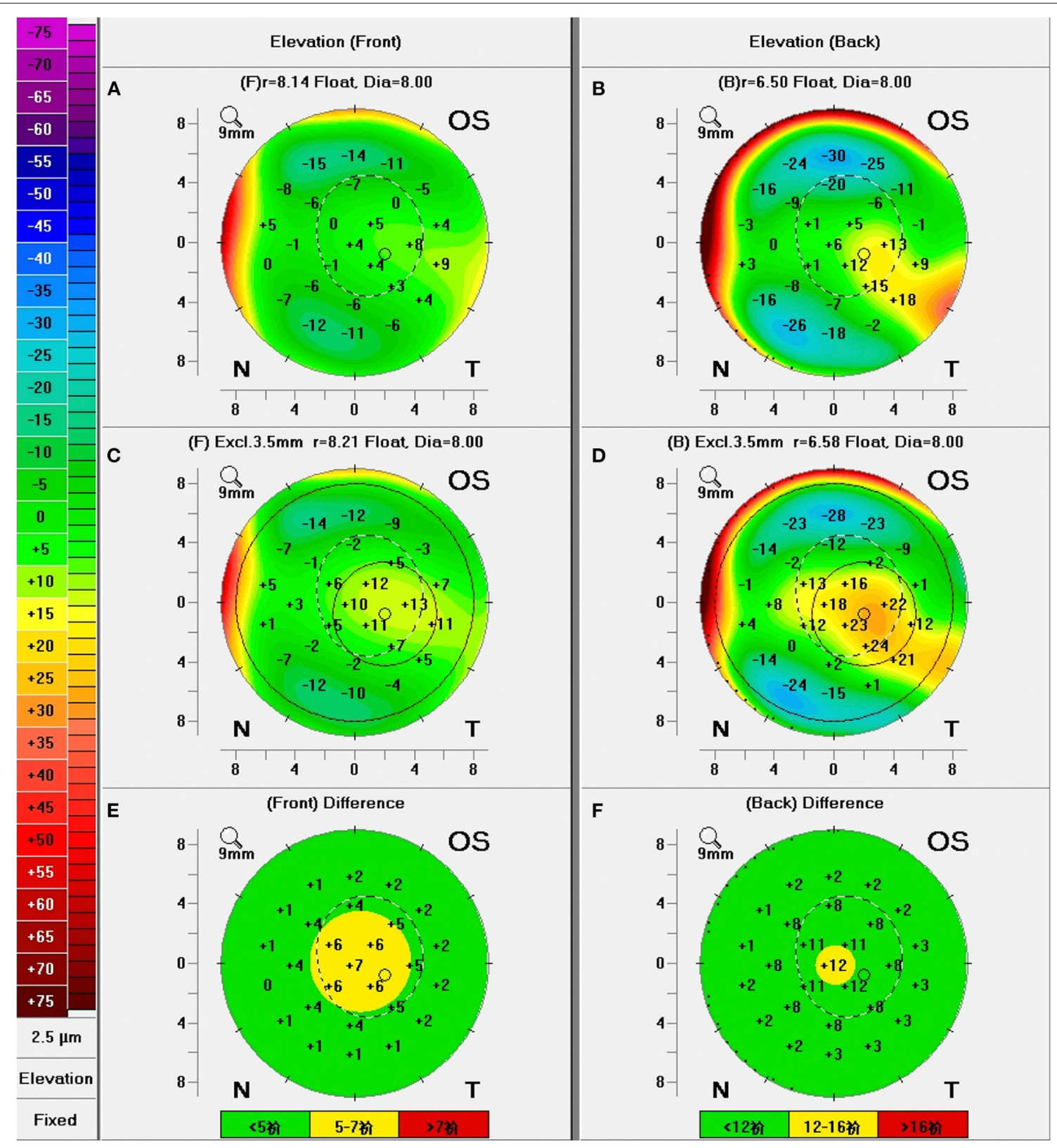

FIGURE 1 | A representative example of elevation maps in patients with allergic conjunctivitis measured by Pentacam. (A,B) Anterior and posterior corneal elevations correlated to the standard best-fit sphere (BFS) reference surfaces; (C,D) Anterior and posterior corneal elevations correlated to the enhanced BFS reference surfaces (with exclusion of a 3.5-mm optical zone in the thinnest portion of the cornea); (E,F) Anterior and posterior corneal elevation differences between the elevation values correlated to the standard BFS and the enhanced BFS.

The typical corneal topography characteristics in $\mathrm{KC}$ patients include increased corneal curvature and corneal thinning. Barreto and colleagues (20) reported a significantly greater central curvature, thinner corneal thickness and higher pachymetric index in VKC patients than in normal subjects. However, contrary to the findings by Barreto et al., our study did not find significant differences in anterior and posterior corneal surface curvature parameters or pachymetric parameters between the normal and AC groups. There are controversial findings in different studies. Similar to our results, no significant change in $\mathrm{K} 1, \mathrm{~K} 2$ or Kmean was found in VKC patients in Ekinci's research (26). The difference in the patient characteristics might be the reason for the different results of the two studies. Barreto's study included only active VKC patients, while our study also included patients with SAC and PAC. VKC, as a more severe form of AC, was reported to have higher levels of ocular inflammatory cytokines and active proteases than SAC and PAC $(27,28)$, 
TABLE 1 | Demographics of the normal and AC groups.

\begin{tabular}{lccc}
\hline Characteristics & $\begin{array}{c}\text { Normal } \\
(\boldsymbol{n}=\mathbf{2 0})\end{array}$ & $\begin{array}{c}\text { AC } \\
(\boldsymbol{n}=\mathbf{3 0})\end{array}$ & $\boldsymbol{p}$ \\
\hline Age, mean \pm SD & $18.75 \pm 7.99$ & $19.37 \pm 10.59$ & 0.83 \\
Sex, male, $n(\%)$ & $13(65.0 \%)$ & $20(66.7 \%)$ & 0.90 \\
IOP (mmHg) & $16.03 \pm 2.40$ & $15.90 \pm 1.96$ & 0.84 \\
S (D) & $-1.92 \pm 1.91$ & $-0.79 \pm 1.55$ & 0.14 \\
C (D) & $-0.50 \pm 0.45$ & $-0.96 \pm 1.29$ & 0.18 \\
SE (D) & $-2.22 \pm 2.07$ & $-1.28 \pm 2.65$ & 0.24 \\
\hline
\end{tabular}

$A C$, allergic conjunctivitis; IOP, intraocular pressure, measured by Corvis ST; S, spherical power; $C$, cylinder power; $S E$, spherical equivalent; $D$, diopter; $S D$, standard deviation.

TABLE 2 | Comparison of corneal topographic parameters between the normal and AC groups.

\begin{tabular}{lccc}
\hline Parameter & $\begin{array}{c}\text { Normal } \\
\text { Mean } \pm \text { SD }\end{array}$ & $\begin{array}{c}\text { AC } \\
\text { Mean } \pm \text { SD }\end{array}$ & $p$ \\
\hline Anterior corneal surface & & & \\
K1 (D) & $42.41 \pm 1.03$ & $42.52 \pm 1.28$ & 0.75 \\
K2 (D) & $43.41 \pm 1.05$ & $43.87 \pm 1.45$ & 0.23 \\
Kmean (D) & $42.90 \pm 1.01$ & $43.20 \pm 1.31$ & 0.38 \\
Kmax (D) & $43.95 \pm 1.13$ & $44.46 \pm 1.50$ & 0.20 \\
Astigmatism (D) & $1.00 \pm 0.54$ & $1.34 \pm 0.84$ & 0.10 \\
Q & $-0.33 \pm 0.11$ & $-0.45 \pm 0.26$ & 0.05 \\
Posterior corneal surface & & & \\
K1 (D) & $-6.08 \pm 0.16$ & $-6.11 \pm 0.21$ & 0.59 \\
K2 (D) & $-6.40 \pm 0.16$ & $-6.5 \pm 0.28$ & 0.15 \\
Kmean (D) & $-6.23 \pm 0.15$ & $-6.29 \pm 0.22$ & 0.25 \\
Astigmatism (D) & $0.29 \pm 0.12$ & $0.38 \pm 0.16$ & 0.02 \\
Q & $-0.26 \pm 0.10$ & $-0.34 \pm 0.11$ & 0.01 \\
\hline AC, & & & \\
\hline
\end{tabular}

$A C$, allergic conjunctivitis; $K 1$, keratometry of the flattest meridian; $K 2$, keratometry of the steepest meridian; Kmean, mean central keratometry; Kmax, maximum keratometry; $Q$, $Q$ value; $D$, diopter; $S D$, standard deviation.

*Statistically significant $(p<0.05)$.

which in turn could induce more severe ocular microstructure damage, indicated by a thinner corneal thickness and greater corneal curvature.

The corneal Q value is a parameter that reflects the corneal shape and represents the degree of corneal asphericity. A more negative $\mathrm{Q}$ value of the posterior corneal surface has been reported in early $\mathrm{KC}$ (15). In this study, we found greater astigmatism and a more negative $\mathrm{Q}$ value for the posterior corneal surface in the AC group than in the normal group. Our findings were consistent with what was reported by Dantas et al. (7). In their study, the $\mathrm{Q}$ value of the cornea was significantly more negative in the VKC group than in the normal group. A more negative $\mathrm{Q}$ value refers to a more prolate shape, with a steeper central cornea and flatter periphery. A previous study reported significant correlations between posterior elevation parameters and posterior $\mathrm{Q}$ values as well as posterior aberrations; hence, the authors speculated that the changes in posterior $\mathrm{Q}$ values and posterior corneal aberrations
TABLE 3 | Comparison of corneal pachymetric parameters between the normal and $\mathrm{AC}$ groups.

\begin{tabular}{lccc}
\hline Parameter & $\begin{array}{c}\text { Normal } \\
\text { Mean } \pm \text { SD }\end{array}$ & $\begin{array}{c}\text { AC } \\
\text { Mean } \pm \text { SD }\end{array}$ & $\boldsymbol{p}$ \\
\hline CCT $(\mu \mathrm{m})$ & $550.15 \pm 21.33$ & $554.10 \pm 24.59$ & 0.56 \\
TCT $(\mu \mathrm{m})$ & $547.35 \pm 23.45$ & $550.43 \pm 25.72$ & 0.75 \\
PImin & $0.73 \pm 0.11$ & $0.73 \pm 0.11$ & 0.82 \\
PImax & $1.28 \pm 0.10$ & $1.36 \pm 0.19$ & 0.09 \\
Plavg & $1.03 \pm 0.09$ & $1.05 \pm 0.12$ & 0.61 \\
ARTmin & $761.30 \pm 124.35$ & $776.30 \pm 150.19$ & 0.71 \\
ARTmax & $429.55 \pm 45.79$ & $415.97 \pm 72.42$ & 0.46 \\
ARTavg & $533.65 \pm 58.67$ & $533.07 \pm 80.80$ & 0.94 \\
\hline
\end{tabular}

AC, allergic conjunctivitis; CCT, central corneal thickness; TCT, thinnest corneal thickness; $\mathrm{PI}$, pachymetric progression index; ART, Ambrosio relational thickness; min, minimum; max, maximum; avg, average; SD, standard deviation.

TABLE 4 | Comparison of corneal elevation parameters between the normal and AC groups.

\begin{tabular}{lccc}
\hline Parameter & $\begin{array}{c}\text { Normal } \\
\text { Mean } \pm \text { SD }\end{array}$ & $\begin{array}{c}\text { AC } \\
\text { Mean } \pm \text { SD }\end{array}$ & p \\
\hline Anterior corneal surface & & \\
TE $(\mu \mathrm{m})$ & $2.80 \pm 1.24$ & $4.07 \pm 2.49$ & $0.04^{*}$ \\
CE $(\mu \mathrm{m})$ & $2.05 \pm 0.94$ & $3.33 \pm 2.45$ & $0.03^{*}$ \\
ME $(\mu \mathrm{m})$ & $0.12 \pm 0.31$ & $0.18 \pm 0.60$ & 0.67 \\
TED $(\mu \mathrm{m})$ & $3.40 \pm 1.10$ & $4.67 \pm 2.22$ & $0.02^{*}$ \\
CED $(\mu \mathrm{m})$ & $3.50 \pm 1.10$ & $4.73 \pm 2.23$ & $0.03^{*}$ \\
MED $(\mu \mathrm{m})$ & $2.60 \pm 0.79$ & $3.64 \pm 1.69$ & $0.01^{*}$ \\
Posterior corneal surface & & \\
TE $(\mu \mathrm{m})$ & $5.00 \pm 2.70$ & $7.73 \pm 3.23$ & $<0.01^{*}$ \\
CE $(\mu \mathrm{m})$ & $0.35 \pm 2.06$ & $1.90 \pm 2.64$ & $0.03^{*}$ \\
ME $(\mu \mathrm{m})$ & $0.56 \pm 0.64$ & $0.48 \pm 0.75$ & 0.69 \\
TED $(\mu \mathrm{m})$ & $3.90 \pm 2.36$ & $6.13 \pm 2.91$ & $0.01^{*}$ \\
CED $(\mu \mathrm{m})$ & $4.00 \pm 2.70$ & $6.57 \pm 3.08$ & $<0.01^{*}$ \\
MED $(\mu \mathrm{m})$ & $2.99 \pm 2.01$ & $4.95 \pm 2.29$ & $<0.01^{*}$ \\
& & &
\end{tabular}

$A C$, allergic conjunctivitis; TE, elevation at the thinnest location; CE, elevation at the central location; $M E$, mean value of elevation in the central $4 \mathrm{~mm}$ zoom; $E D$, elevation difference; $S D$, standard deviation.

* Statistically significant $(p<0.05)$.

were attributed to alterations in corneal posterior elevation (15). Thus, we hypothesize that the more negative $\mathrm{Q}$ value and greater astigmatism of the corneal posterior surface in AC patients might also be induced by alterations in corneal posterior elevation.

Previous studies reported that corneal anterior and posterior elevation values were significantly higher in early KC patients than in normal subjects $(10,11,13)$. Our study also found much higher corneal elevation values or elevation differences in either the anterior or posterior surface in AC patients than in normal subjects. However, whether KC-like changes in corneal elevation could predict $\mathrm{KC}$ development in $\mathrm{AC}$ patients still requires further investigation. Moreover, several studies have shown that 
TABLE 5 | Correlation of altered corneal elevation parameters with eye rubbing frequency and ocular allergy sign severity in AC patients.

\begin{tabular}{|c|c|c|c|c|c|c|c|c|c|c|c|}
\hline & & \multicolumn{5}{|c|}{ Anterior corneal surface } & \multicolumn{5}{|c|}{ Posterior corneal surface } \\
\hline & & TE & CE & TED & CED & MED & TE & CE & TED & CED & MED \\
\hline \multirow[t]{2}{*}{ Eye rubbing } & r & 0.251 & 0.414 & 0.424 & 0.452 & 0.462 & 0.277 & 0.023 & 0.247 & 0.226 & 0.211 \\
\hline & $p$ & 0.181 & $0.023^{\star}$ & $0.020^{\star}$ & $0.012^{\star}$ & $0.010^{\star}$ & 0.138 & 0.903 & 0.188 & 0.230 & 0.264 \\
\hline \multirow[t]{2}{*}{ Hyperemia } & r & 0.011 & 0.082 & 0.099 & 0.109 & 0.077 & -0.056 & 0.039 & -0.221 & -0.230 & -0.256 \\
\hline & $p$ & 0.956 & 0.666 & 0.601 & 0.567 & 0.685 & 0.769 & 0.836 & 0.242 & 0.222 & 0.173 \\
\hline \multirow[t]{2}{*}{ Swelling } & r & 0.257 & 0.312 & 0.403 & 0.420 & 0.409 & 0.075 & 0.024 & -0.028 & -0.075 & -0.090 \\
\hline & $p$ & 0.170 & 0.093 & $0.027^{\star}$ & $0.021^{\star}$ & $0.025^{\star}$ & 0.692 & 0.900 & 0.885 & 0.693 & 0.635 \\
\hline \multirow[t]{2}{*}{ Papillae } & $r$ & 0.280 & 0.318 & 0.455 & 0.455 & 0.452 & 0.103 & 0.141 & 0.008 & -0.039 & -0.024 \\
\hline & $p$ & 0.135 & 0.087 & $0.011^{\star}$ & $0.011^{\star}$ & $0.012^{*}$ & 0.588 & 0.458 & 0.967 & 0.838 & 0.899 \\
\hline \multirow[t]{2}{*}{ Epithelial disorder } & $r$ & 0.200 & 0.465 & 0.459 & 0.443 & 0.449 & 0.009 & 0.026 & -0.035 & -0.066 & -0.070 \\
\hline & $p$ & 0.289 & $0.010^{\star}$ & $0.011^{\star}$ & $0.014^{\star}$ & $0.013^{\star}$ & 0.963 & 0.890 & 0.854 & 0.730 & 0.714 \\
\hline
\end{tabular}

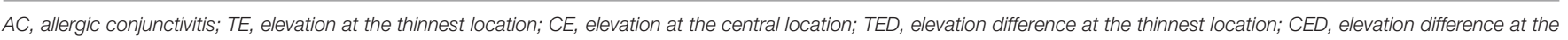
central location; MED, mean value of elevation difference in the central $4 \mathrm{~mm}$ zoom.

*Statistically significant $(p<0.05)$.

TABLE 6 | Correlation of corneal elevation parameters with TBI in AC patients.

\begin{tabular}{|c|c|c|c|c|c|c|c|c|c|c|c|}
\hline & & \multicolumn{5}{|c|}{ Anterior corneal surface } & \multicolumn{5}{|c|}{ Posterior corneal surface } \\
\hline & & TE & CE & TED & CED & MED & TE & CE & TED & CED & MED \\
\hline \multirow[t]{2}{*}{ TBI } & r & 0.608 & 0.464 & 0.754 & 0.727 & 0.750 & 0.563 & 0.156 & 0.341 & 0.318 & 0.323 \\
\hline & $p$ & $<0.001^{*}$ & $0.010^{*}$ & $<0.001^{\star}$ & $<0.001^{\star}$ & $<0.001^{*}$ & $0.001^{\star}$ & 0.412 & 0.065 & 0.087 & 0.081 \\
\hline
\end{tabular}

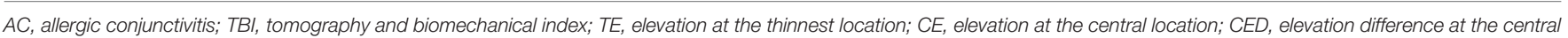
location; MED, mean value of elevation difference in the central $4 \mathrm{~mm}$ zone.

${ }^{\star}$ Statistically significant $(p<0.05)$.

posterior elevation parameters are more sensitive than anterior elevation parameters in differentiating $\mathrm{KC}$ from normal eyes $(10,12,16)$. For example, Huseynli et al. (10) further showed that among the parameters measured by Pentacam, including corneal topographic parameters, pachymetric parameters, elevation parameters and topometric parameters, anterior and posterior elevation showed the highest AUROCs (0.935 and 0.897, respectively) to differentiate early $\mathrm{KC}$ patients from normal subjects. Therefore, among the parameters evaluated in their study, posterior corneal elevation changes were identified as the earliest sign of subclinical $\operatorname{KC}(15,16)$. Additionally, in our study, we found that the increased elevations of both the anterior and posterior corneal surfaces were positively correlated with altered TBI, which was identified as a sensitive indicator of $\mathrm{KC}$ development risk in $\mathrm{AC}$ patients in our previous study (19). This result to a certain extent demonstrates the possibility and risk of $\mathrm{KC}$ occurrence in $\mathrm{AC}$ patients. In addition, our findings indicate that corneal elevation changes occurred earlier than the occurrence of abnormal corneal pachymetric distribution and increased corneal curvature in AC patients. Therefore, measurement of corneal elevation, especially of the posterior surface, may aid in evaluating the risk of $\mathrm{KC}$ development in AC patients. However, except for $\mathrm{KC}$, higher corneal elevations have also been reported in other diseases such as patients with Familial Mediterranean fever (29). Even some normal subjects may have abnormal corneal elevations indicated by yellow color in the Belin/Ambrósio Enhanced Ectasia Display. Thus, it would be better to combine corneal tomography with other $\mathrm{KC}$ detection tools such as corneal biomechanical assessment and corneal epithelial thickness measurement to comprehensively evaluate the $\mathrm{KC}$ risk in AC patients.

Furthermore, we found that altered anterior corneal elevation was positively correlated with eye rubbing frequency and ocular allergy sign severity in AC patients. However, there was no significant correlation between posterior surface corneal elevation and ocular allergy sign severity. The anterior surface of the cornea could be affected by various factors. For example, AC patients often have unstable tear film and dry eye (30-33). Irregular tear films and the use of artificial tears in AC patients are factors influencing the corneal anterior surface $(11,26)$. Moreover, the corneal epithelial damage and thinning caused by excessive eye rubbing in $\mathrm{AC}$ patients could also induce increased irregularity of the anterior corneal surface. Therefore, anterior corneal elevation may not be suitable for use as an indicator of $\mathrm{KC}$ risk in $\mathrm{AC}$ patients due to its vulnerability to multiple factors, including ocular surface inflammation conditions, tear film stability and corneal epithelial defects.

Contrary to anterior corneal surface measurements, posterior corneal surface measurements were not influenced by the irregularity and allergic signs of the ocular surface. Although 
there was no significant correlation between altered posterior elevation and the severity of ocular allergy signs in AC patients, the alterations in posterior elevation were not necessarily independent of the inflammatory condition and the course of ocular allergy. Increased release of inflammatory cytokines, including matrix metalloproteinase (MMP)-1,-3,-7, and -13 , interleukin (IL) $-4,-5,-6$, and -8 and tumor necrosis factor (TNF)- $\alpha$ and $-\beta$, induced by excessive eye rubbing in $\mathrm{AC}$ patients was reported to mediate pathological apoptosis of keratocytes and corneal fibroblasts, which could further induce alterations in the posterior corneal surface and corneal weakness (27, 34-36). Thus, posterior elevation parameters, which were mainly affected by corneal chronic and long-term inflammation, could be used as sensitive and stable indicators of $\mathrm{KC}$ risk in AC patients.

A limitation of this study is the small sample size; thus, studies with a larger sample size are needed to compare corneal tomography changes among different types of AC. Moreover, a follow-up study to explore corneal elevation changes over time is necessary.

In conclusion, compared to normal subjects, AC patients carry an increased risk of corneal ectasia, as indicated by higher corneal elevation parameters. Posterior corneal elevation parameters could be used as sensitive and stable indicators of $\mathrm{KC}$ risk in AC patients.

\section{REFERENCES}

1. Aydin E, Gokhale M, Azizoglu S, Suphioglu C. To see or not to see: a systematic review of the importance of human ocular surface cytokine biosignatures in ocular allergy. Cells. (2019) 8:620. doi: 10.3390/cells8060620

2. Ahmed AS, El-Agha MSH, Khaled MO, Shousha SM. The prevalence of keratoconus in children with allergic eye disease in an Egyptian population. Eur J Ophthalmol. (2020) 31:1571-76. doi: 10.1177/1120672120942691

3. Moran S, Gomez L, Zuber K, Gatinel D. A case-control study of keratoconus risk factors. Cornea. (2020) 39:697-701. doi: 10.1097/ICO.0000000000002283

4. Lee HK, Jung EH, Cho BJ. Epidemiological association between systemic diseases and keratoconus in a korean population: a 10-year nationwide cohort study. Cornea. (2020) 39:348-53. doi: 10.1097/ICO.0000000000002206

5. Sharma N, Rao K, Maharana PK, Vajpayee RB. Ocular allergy and keratoconus. Indian J Ophthalmol. (2013) 61:4079. doi: 10.4103/0301-4738.116063

6. Ben-Eli H, Erdinest N, Solomon A. Pathogenesis and complications of chronic eye rubbing in ocular allergy. Curr Opin Allergy Clin Immunol. (2019) 19:52634. doi: 10.1097/ACI.0000000000000571

7. Correa Dantas PE, Alves MR, Nishiwaki-Dantas MC. Topographic corneal changes in patients with vernal keratoconjunctivitis. Arq Bras Oftalmol. (2005) 68:593-8. doi: 10.1590/S0004-27492005000500004

8. Kataria P, Padmanabhan P, Gopalakrishnan A, Padmanaban V, Mahadik S, Ambrósio R. Accuracy of scheimpflug-derived corneal biomechanical and tomographic indices for detecting subclinical and mild keratectasia in a South Asian population. J Cataract Refract Surg. (2019) 45:32836. doi: 10.1016/j.jcrs.2018.10.030

9. Golan O, Hwang ES, Lang P, Santhiago MR, Abulafia A, Touboul $\mathrm{D}$, et al. Differences in posterior corneal features between normal corneas and subclinical keratoconus. J Refract Surg. (2018) 34:66470. doi: 10.3928/1081597X-20180823-02

10. Huseynli S, Salgado-Borges J, Alio JL. Comparative evaluation of scheimpflug tomography parameters between thin non-keratoconic, subclinical

\section{DATA AVAILABILITY STATEMENT}

The raw data supporting the conclusions of this article will be made available by the authors, without undue reservation.

\section{ETHICS STATEMENT}

The studies involving human participants were reviewed and approved by the Ethics Committee of Zhongshan Ophthalmic Center, Sun Yat-sen University (2020KYPJ008). Written informed consent to participate in this study was provided by the participants' legal guardian/next of kin.

\section{AUTHOR CONTRIBUTIONS}

JY and SL designed and supervised the study. QW and FY analyzed and interpreted the data and prepared the manuscript. $\mathrm{ZF}, \mathrm{WL}, \mathrm{NL}, \mathrm{XD}$, and $\mathrm{XZ}$ contributed to data collection. All authors contributed to the article and approved the submitted version.

\section{FUNDING}

This work was supported by grants from the National Natural Science Foundation of China (82171015).

keratoconic, and mild keratoconic corneas. Eur J Ophthalmol. (2018) 28:521-34. doi: 10.1177/1120672118760146

11. Miháltz K, Kovács I, Takács Á, Nagy ZZ. Evaluation of keratometric, pachymetric, and elevation parameters of keratoconic corneas with pentacam. Cornea. (2009) 28:976-80. doi: 10.1097/ICO.0b013e31819e34de

12. Muftuoglu O, Ayar O, Hurmeric V, Orucoglu F, Kilic I. Comparison of multimetric D index with keratometric, pachymetric, and posterior elevation parameters in diagnosing subclinical keratoconus in fellow eyes of asymmetric keratoconus patients. J Cataract Refract Surg. (2015) 41:55765. doi: $10.1016 /$ j.jcrs.2014.05.052

13. Kamiya K, Ishii R, Shimizu K, Igarashi A. Evaluation of corneal elevation, pachymetry and keratometry in keratoconic eyes with respect to the stage of Amsler-Krumeich classification. Br J Ophthalmol. (2014) 98:45963. doi: 10.1136/bjophthalmol-2013-304132

14. de Sanctis U, Loiacono C, Richiardi L, Turco D, Mutani B, Grignolo FM. Sensitivity and specificity of posterior corneal elevation measured by pentacam in discriminating keratoconus/subclinical keratoconus. Ophthalmology. (2008) 115:1534-9. doi: 10.1016/j.ophtha.2008.02.020

15. Elkitkat RS, Gharieb HM, Othman IS. Accuracy of the posterior corneal elevation values of pentacam HR from different reference surfaces in early ectasia diagnosis. Int Ophthalmol. (2021) 41:629-38. doi: 10.1007/s10792-020-01618-8

16. Muftuoglu O, Ayar O, Ozulken K, Ozyol E, Akinci A. Posterior corneal elevation and back difference corneal elevation in diagnosing forme fruste keratoconus in the fellow eyes of unilateral keratoconus patients. J Cataract Refract Surg. (2013) 39:1348-57. doi: 10.1016/j.jcrs.2013.03.023

17. Gautam V, Chaudhary M, Sharma AK, Shrestha GS, Rai PG. Topographic corneal changes in children with vernal keratoconjunctivitis: a report from Kathmandu, Nepal. Contact Lens Anterior Eye. (2015) 38:4615. doi: 10.1016/j.clae.2015.05.013

18. Emre S, Başer E, Öztürk B, Zorlu S, Uzun Ö, Gülhan C. Corneal biochemical features of patients with vernal keratoconjunctivitis. Graefe's Arch Clin Exp Ophthalmol. (2013) 251:555-8. doi: 10.1007/s00417-012-2089-2 
19. Wang Q, Deng Y, Li S, Du X, Zhao X, Zhang T, et al. Corneal biomechanical changes in allergic conjunctivitis. Eye Vis. (2021) 8:19. doi: 10.1186/s40662-021-00241-7

20. Barreto J, Netto MV, Santo RM, José NK, Bechara SJ. Slit-scanning topography in vernal keratoconjunctivitis. Am J Ophthalmol. (2007) 143:2504. doi: 10.1016/j.ajo.2006.10.027

21. de Sanctis U, Missolungi A, Mutani B, Richiardi L, Grignolo FM. Reproducibility and repeatability of central corneal thickness measurement in keratoconus using the rotating scheimpflug camera and ultrasound pachymetry. Am J Ophthalmol. (2007) 144:712-718. doi: 10.1016/j.ajo.2007.07.021

22. Chen D, Lam AKC. Intrasession and intersession repeatability of the pentacam system on posterior corneal assessment in the normal human eye. J Cataract Refract Surg. (2007) 33:448-54. doi: 10.1016/j.jcrs.2006.11.008

23. Mazzotta C, Traversi C, Mellace P, Bagaglia SA, Zuccarini S, Mencucci R, et al. Keratoconus progression in patients with allergy and elevated surface matrix metalloproteinase 9 point-of-care test. Eye Contact Lens. (2018) 44:S48S53. doi: 10.1097/ICL.0000000000000432

24. Cingu AK, Cinar Y, Turkcu FM, Sahin A, Ari S, Yuksel H, et al. Effects of vernal and allergic conjunctivitis on severity of keratoconus. Int J Ophthalmol. (2013) 6:370-4. doi: 10.3980/j.issn.2222-3959.2013.03.21

25. Hashemi H, Heydarian S, Hooshmand E, Saatchi M, Yekta A, Aghamirsalim $\mathrm{M}$, et al. The prevalence and risk factors for keratoconus: a systematic review and meta-analysis. Cornea. (2020) 39:263-70. doi: 10.1097/ICO.0000000000002150

26. EKINCI DY, ALPARSLAN N, CEBECI Z, YAVUZ SARIÇAY L, DÖNMEZ GÜN R. The topographic corneal changes in patients with vernal keratoconjunctivitis. Turkiye Klin J Ophthalmol. (2019) 28:99-104. doi: 10.5336/ophthal.2018-63355

27. Zheng X, Yao J, Li B. Expression of TSLP and downstream molecules IL-4, IL-5, and IL-13 on the eye surface of patients with various types of allergic conjunctivitis. J Ophthalmol. (2016) 2016:5072781. doi: 10.1155/2016/5072781

28. Kumagai N, Yamamoto K, Fukuda K, Nakamura Y, Fujitsu Y, Nuno Y, et al. Active matrix metalloproteinases in the tear fluid of individuals with vernal keratoconjunctivitis. J Allergy Clin Immunol. (2002) 110:48991. doi: 10.1067/mai.2002.126379

29. Kosekahya P, Ucgul Atilgan C, Atilgan KG, Koc M, Tekin K, Caglayan M, et al. Increased corneal ectasia risk in patients with familial mediterranean fever. Curr Eye Res. (2019) 44:351-5. doi: 10.1080/02713683.2018.1554150

30. Hom MM, Nguyen AL, Bielory L. Allergic conjunctivitis and dry eye syndrome. Ann Allergy Asthma Immunol. (2012) 108:163-6. doi: 10.1016/j.anai.2012.01.006
31. Kim M, Oh JH, Park CY, Lee SW. Dry eye disease and allergic conditions: a Korean nationwide population-based study. Am J Rhinol Allergy. (2016) 30:397-401. doi: 10.2500/ajra.2016.30.4361

32. Dogru M, Gunay M, Celik G, Aktas A. Evaluation of the tear film instability in children with allergic diseases. Cutan Ocul Toxicol. (2016) 35:49-52. doi: 10.3109/15569527.2015.1010727

33. Villani E, Rabbiolo G, Nucci P. Ocular allergy as a risk factor for dry eye in adults and children. Curr Opin Allergy Clin Immunol. (2018) 18:398403. doi: 10.1097/ACI.0000000000000471

34. Leonardi A, Curnow SJ, Zhan H, Calder VL. Multiple cytokines in human tear specimens in seasonal and chronic allergic eye disease and in conjunctival fibroblast cultures. $\mathrm{Cl}$ in Exp Allergy. (2006) 36:777-84. doi: 10.1111/j.1365-2222.2006.02499.x

35. Uchio E, Ono SY, Ikezawa Z, Ohno S. Tear Levels of Interferon$\gamma$, Interleukin (IL)-2, IL-4 and IL-5 in Patients With Vernal Keratoconjunctivitis, Atopic Keratoconjunctivitis and Allergic Conjunctivitis. Clin Exp Allergy. (2000) 30:103-9. doi: 10.1046/j.1365-2222.2000. 00699.x

36. Leonardi A, Sathe S, Bortolotti M, Beaton A, Sack R. Cytokines, matrix metalloproteases, angiogenic and growth factors in tears of normal subjects and vernal keratoconjunctivitis patients. Allergy Eur J Allergy Clin Immunol. (2009) 64:710-7. doi: 10.1111/j.1398-9995.2008.01 $858 . \mathrm{x}$

Conflict of Interest: The authors declare that the research was conducted in the absence of any commercial or financial relationships that could be construed as a potential conflict of interest.

Publisher's Note: All claims expressed in this article are solely those of the authors and do not necessarily represent those of their affiliated organizations, or those of the publisher, the editors and the reviewers. Any product that may be evaluated in this article, or claim that may be made by its manufacturer, is not guaranteed or endorsed by the publisher.

Copyright (c) 2021 Wang, Yu, Feng, Li, Li, Du, Zhao, Li and Yuan. This is an open-access article distributed under the terms of the Creative Commons Attribution License (CC BY). The use, distribution or reproduction in other forums is permitted, provided the original author(s) and the copyright owner(s) are credited and that the original publication in this journal is cited, in accordance with accepted academic practice. No use, distribution or reproduction is permitted which does not comply with these terms. 